\title{
Computed Tomography of the Abdomen with Contrast
}

National Cancer Institute

\section{Source}

National Cancer Institute. Computed Tomography of the Abdomen with Contrast. NCI

Thesaurus. Code C137878.

Computed tomography of the abdomen with the use of a contrast agent to enhance the image. 